\title{
Ion dynamics during compression of Mercury's magnetosphere
}

\author{
D. C. Delcourt ${ }^{1}$, T. E. Moore ${ }^{2}$, and M.-C. H. Fok ${ }^{2}$ \\ ${ }^{1}$ LPP, Ecole Polytechnique-CNRS-UPMC, UMR7648, Saint-Maur des Fossés, France \\ ${ }^{2}$ NASA, Goddard Space Flight Center, Greenbelt, Maryland, USA
}

Received: 17 February 2010 - Revised: 28 June 2010 - Accepted: 14 July 2010 - Published: 3 August 2010

\begin{abstract}
Because of the small planetary magnetic field as well as proximity to the Sun that leads to enhanced solar wind pressure as compared to Earth, the magnetosphere of Mercury is very dynamical and at times subjected to prominent compression. We investigate the dynamics of magnetospheric ions during such compression events. Using threedimensional single-particle simulations, we show that the electric field induced by the time varying magnetic field can lead to significant ion energization, up to several hundreds of eVs or a few keVs. This energization occurs in a nonadiabatic manner, being characterized by large enhancements of the ion magnetic moment and bunching in gyration phase. It is obtained when the ion cyclotron period is comparable to the field variation time scale. This condition for nonadiabatic heating is realized in distinct regions of space for ions with different mass-to-charge ratios. During compression of Mercury's magnetosphere, heavy ions originating from the planetary exosphere may be subjected to such an abrupt energization, leading to loading of the magnetospheric lobes with energetic material.
\end{abstract}

Keywords. Magnetospheric physics

(Planetary magnetospheres)

\section{Introduction}

The early measurements of Mariner-10 in 1974-1975 revealed that Mercury possesses a weak intrinsic magnetic field that leads to the formation of a miniature magnetosphere (e.g., Ness, 1979) with typical time scales much smaller (by about a factor 20) than those at Earth (e.g., Russell and Walker, 1985). The recent observations of MESSENGER during flybys of Mercury confirm this result, the internal field being in first approximation dipolar with an estimated

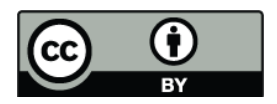

Correspondence to: D. C. Delcourt (dominique.delcourt@1pp.polytechnique.fr) moment of $\sim 250 \mathrm{nT} R_{\mathrm{M}}^{3}$ ( $R_{\mathrm{M}}$ denoting Mercury's radius) (Anderson et al., 2008). These observations also provided evidences of a substantial contribution of ionized material originating from the planetary exosphere (e.g., $\mathrm{Na}^{+}-\mathrm{Mg}^{+}$, $\mathrm{S}^{+}-\mathrm{O}_{2}^{+}, \mathrm{K}^{+}-\mathrm{Ca}^{+}$) to the inner magnetotail (Zurbuchen et al., 2008). Because of the small characteristic length scales of the hermean magnetosphere, kinetic processes that follow from finite Larmor radii of the particles are expected to play a significant role (e.g., Slavin et al., 2009). Also, given the small magnitude of the internal field and the enhanced solar wind pressure due to proximity with the Sun, the magnetosphere of Mercury is expected to be very dynamical and subjected to frequent reconfigurations. As a matter of fact, modeling studies for large solar wind speed and density reported the possibility of extreme magnetospheric compression such that the magnetopause is pushed down to the planet surface (e.g., Kabin et al., 2000); hence, a direct interaction between this surface and the solar wind. In the present study, we examine the dynamics of magnetospheric ions during such compression events. We show that, for ion species with cyclotron period comparable to the field variation time scale, prominent nonadiabatic heating may be achieved under the effect of the electric field induced by the magnetic field reconfiguration.

\section{Modeling of magnetic field reconfiguration}

Due to the combination of a weak planetary magnetic field (about 1000 times smaller than that of Earth) and a large solar wind pressure (about 10 times larger than that at Earth), Mercury's magnetosphere is expected to be fairly dynamical. According to modeling studies (e.g., Kabin et al., 2000; Benna et al., 2010), the subsolar magnetopause is expected to be located at a radial distance of 1.5 to $2 R_{\mathrm{M}}$, although recent measurements from MESSENGER suggest a complex situation with a double magnetopause signature in the dawn region (Slavin et al., 2008). The location of the magnetopause

Published by Copernicus Publications on behalf of the European Geosciences Union. 
depends upon the characteristics of the solar wind as well as those of the internally generated magnetic field (see, e.g., Kabin et al., 2008). At times, it may significantly deviate from the above mean value and be shifted toward the planet. In this regard, Kabin et al. (2000) demonstrated that, during events of enhanced (by about a factor 9 as compared to the case with nominal Parker spiral) solar wind dynamical pressure, the magnetopause may be pushed below $1 R_{\mathrm{M}}$ radial distance, leading to the formation of a "bald spot" around the subsolar point and paving the way for direct interaction between the solar wind and the planet surface. The purpose of the present study is to examine the behavior of magnetospheric ions during such compression events, though not as extreme as the above "bald spot" case. In the same vein, at Earth, Cladis et al. (2000) examined the transport of ions in the high-altitude polar region in response to a sudden compression of the magnetosphere caused by a coronal mass ejection. In this latter event, because of a solar wind pressure increase by about a factor 7, cusp field lines were rapidly brought close to the Polar spacecraft, leading to abrupt energization of the ions measured by the TIMAS instrument. The study of Cladis et al. (2000) suggested that this energization follows from centrifugal acceleration in the parallel direction in the course of the short-lived antisunward transport. This short-lived transport has characteristic scales of $\sim 2 R_{\mathrm{E}}$ in 2 min (i.e., an average speed of $\sim 100 \mathrm{~km} / \mathrm{s}$ ) at Polar altitude.

To investigate the behavior of ions during compression of Mercury's magnetosphere, we performed single-particle simulations in three-dimensional electric and magnetic fields that provide a first-order description of the hermean environment. As for the magnetic field, it was accounted for using a modified version of the model of Luhmann and Friesen (1979). This simple model that reproduces basic features of the magnetosphere, consists of the superposition of the planetary dipole field and an infinite Harris-type current sheet (see Delcourt et al., 2003). The parameters of this model are the dipole moment $M$, the current sheet half-thickness $L$, and the current sheet field $B_{T}$. Taking $M=250 \mathrm{nT} R_{\mathrm{M}}^{3}$ (e.g., Anderson et al., 2008) and assuming a current sheet thickness of a few thousand of kilometers ( $L$ $=0.5 R_{M}$ ), the magnetic field line geometry is solely controlled by $B_{\mathrm{T}}$. As noted by Luhmann and Friesen (1979), the effects on the magnetosphere of the temporal variability of the solar wind are simply modeled by adjusting this latter parameter $B_{\mathrm{T}}$. In the present study, we simulate the compression of the magnetosphere by increasing $B_{\mathrm{T}}$ (equivalently, by increasing the magnetic flux in the lobes) by a factor 2 , from $B_{\mathrm{T}}=50 \mathrm{nT}$ in the initial state up to $B_{\mathrm{T}}=100 \mathrm{nT}$ in the final state. The magnetospheric configurations obtained before and after compression are illustrated in Fig. 1 that shows magnetic field lines in the noon-midnight meridian plane. In this figure as well as in the following, note that the $\mathrm{X}$ axis points in the antisunward direction, and the $\mathrm{Y}$ axis, from dusk to dawn. It can be seen in Fig. 1 that, in the initial

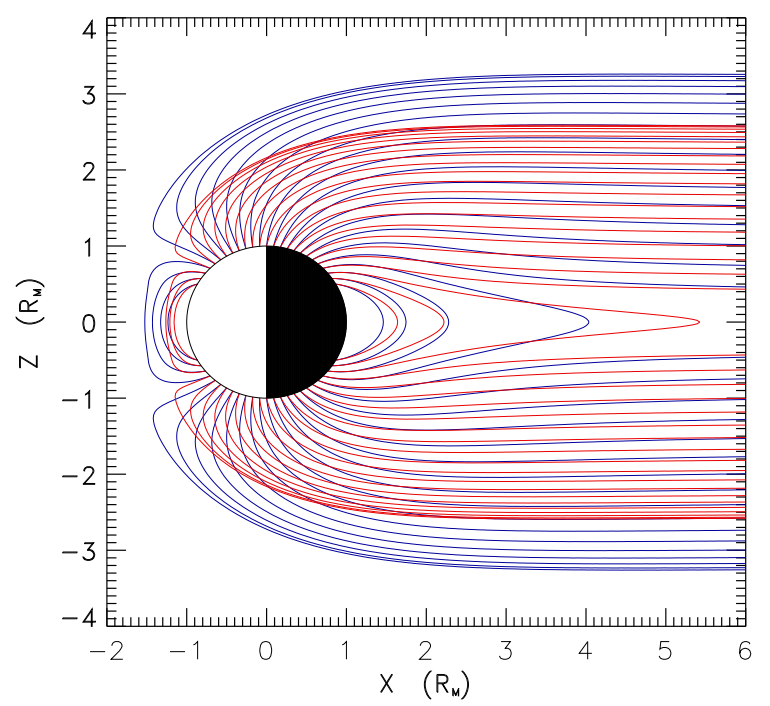

Fig. 1. Magnetic field lines in the noon-midnight meridian plane before and after the reconfiguration (color-coded in blue and red, respectively). The $\mathrm{X}$ axis points toward the tail, the $\mathrm{Y}$ axis, from dusk to dawn.

configuration (blue lines), the subsolar point is located near $\sim 1.5 R_{\mathrm{M}}$. As a result of compression, this subsolar point is shifted down to $\sim 1.2 R_{\mathrm{M}}$ in the final configuration (red lines).

As for the very transition between pre- and postcompression configurations, it was modeled using an approach similar to that of Delcourt et al. (1990). That is, the magnetic field at a given position $\boldsymbol{r}$ and a given time $t$ was obtained as:

$\boldsymbol{B}(\boldsymbol{r}, t)=\boldsymbol{B}_{\mathrm{o}}(\boldsymbol{r})+f(t)\left[\boldsymbol{B}_{\mathrm{f}}(\boldsymbol{r})-\boldsymbol{B}_{\mathrm{o}}(\boldsymbol{r})\right]$

where $\boldsymbol{B}_{\mathrm{o}}$ and $\boldsymbol{B}_{\mathrm{f}}$ correspond to initial and final configurations, respectively. In Eq. (1), $f(t)$ describes the rate of change of the magnetic field. As described in Appendix A of Delcourt et al. (1990), imposing a zero induced electric field as well as its zero slope at the onset and at the end of the magnetic transition leads to a polynomial of degree 5 for $f(t)$. Denoting by $\tau_{\mathrm{B}}$ the time scale of the reconfiguration, this polynomial smoothly varies between 0 at $t=0$ and 1 at $t=\tau_{\mathrm{B}}$.

As for the electric field, it was considered to be the sum of two contributions. The first contribution is that induced by the time varying magnetic field, viz., $\boldsymbol{E}=-\partial \boldsymbol{A} / \partial t$ where the vector potential $\boldsymbol{A}$ is such that $\operatorname{curl}(\boldsymbol{A})=\boldsymbol{B}$ as given by (1). Since the vector potential is undetermined to the gradient of a scalar field, a second contribution $\nabla \Phi$ is considered that cancels the parallel component of the induced electric field, that is, $\boldsymbol{E} \cdot \boldsymbol{B}=0$. The calculation of this second contribution is described in Appendix B of Delcourt et al. (1990). As discussed by Heikkila and Pellinen (1977), this latter contribution actually redistributes the field in the perpendicular 
direction and accounts for plasma polarization. At a given position $\boldsymbol{r}$ and time $t$, the electric field is thus obtained as:

$\boldsymbol{E}(\boldsymbol{r}, t)=-[\partial \boldsymbol{A}(\boldsymbol{r}, t) / \partial t]-\nabla \Phi_{\mathrm{pol}}(\boldsymbol{r}, t)$

with

$\boldsymbol{A}(\boldsymbol{r}, t)=\boldsymbol{A}_{\mathrm{o}}(\boldsymbol{r})+f(t)\left[\boldsymbol{A}_{\mathrm{f}}(\boldsymbol{r})-\boldsymbol{A}_{\mathrm{o}}(\boldsymbol{r})\right]$

and

$\Phi_{\mathrm{pol}}(\boldsymbol{r}, t)=-\int[\partial \boldsymbol{A}(\boldsymbol{r}, t) / \partial t] \cdot \boldsymbol{b}(\boldsymbol{r}, t) d s$

Here, $\boldsymbol{A}_{\mathrm{o}}$ and $\boldsymbol{A}_{\mathrm{f}}$ relate to initial and final configurations, respectively, $\boldsymbol{b}$ is a unit vector in the direction of $\boldsymbol{B}$, and $s$ is the curvilinear abscissa along the field line. Equation (4) shows the general expression of the polarization potential, $\nabla \Phi_{\mathrm{pol}}$ in (2) being obtained via computation of $\Phi_{\mathrm{pol}}$ at $\boldsymbol{r}$ and at two neighboring points, $\boldsymbol{r}_{1}$ and $\boldsymbol{r}_{2}$, such that $\boldsymbol{r}_{1}-\boldsymbol{r}$ and $\boldsymbol{r}_{2}-\boldsymbol{r}$ define two directions perpendicular to each other and perpendicular to $\boldsymbol{B}$.

The electric and magnetic field variations obtained from Eqs. (1-4) can be appreciated in Fig. 2 that shows the evolution of a field line anchored at high latitude in the dayside sector (left panel). In a like manner to Cladis et al. (2000) (see, e.g., their Fig. 3), it can be seen in Fig. 2 that this field line is rapidly brought into the antisunward direction. In this figure as well as in the following simulations, the time scale $\tau_{\mathrm{B}}$ of the magnetic transition was set to $20 \mathrm{~s}$. Because propagation effects are not accounted for in the present model, this $20 \mathrm{~s}$ time scale describes the duration of the local magnetic field reconfiguration, the overall compression of the magnetosphere itself occurring on a significantly larger time scale (a few minutes). To make this more apparent, the black line in the left panel of Fig. 2 shows the trajectory of a zero energy particle that experiences only $\boldsymbol{E} \times \boldsymbol{B}$ drift from initial to final configurations. The right panels of Fig. 2 show the electric field as well as the $\boldsymbol{E} \times \boldsymbol{B}$ drift speed along this path. It can be seen in these panels that our model reconfiguration goes together with a duskward oriented electric field that reaches a peak magnitude of $\sim 7 \mathrm{mV} / \mathrm{m}$. The associated $\boldsymbol{E} \times \boldsymbol{B}$ drift speed peaks at $\sim 80 \mathrm{~km} / \mathrm{s}$, its average value being of the order of $40-50 \mathrm{~km} / \mathrm{s}$, i.e., significantly smaller (by about a factor 2 ) than that reported by Cladis et al. (2000) during compression of the Earth's magnetosphere.

Finally, in addition to the electric field induced by the time-varying magnetic field (see above Eq. 2), a large-scale convection electric field resulting from coupling between the solar wind and the magnetosphere was accounted for in the simulations. This latter electric field was modeled in first approximation using a Volland-Stern description (see, e.g., Volland, 1978) with a two-cell pattern of potential distribution in the immediate vicinity of the planet. This latter potential distribution is characterized by anti-sunward transport at high latitudes (above $45^{\circ}$ invariant latitude) and sunward transport below. An average cross-polar cap potential drop of $20 \mathrm{kV}$ was assumed, leading to a dawn-to-dusk convection electric field of $\sim 2 \mathrm{mV} / \mathrm{m}$ in the central magnetotail (e.g., Ip, 1987).
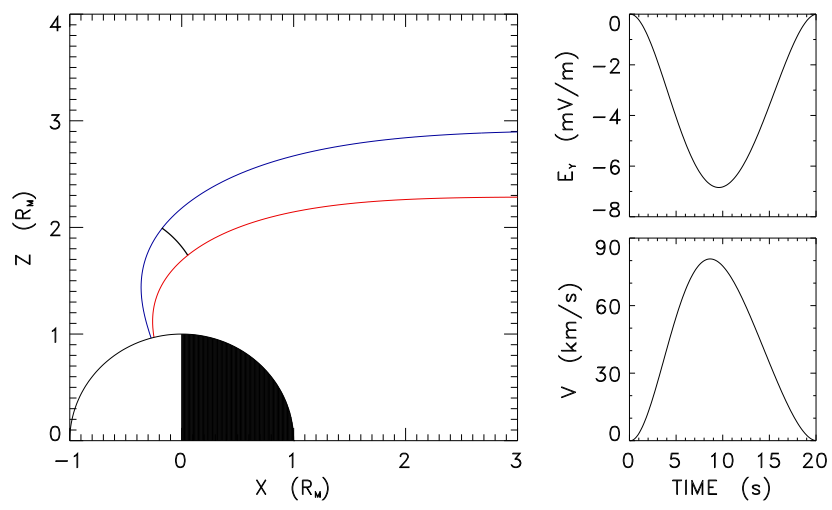

Fig. 2. (Left) Initial and final configurations of a given magnetic field line in the polar region (color-coded in blue and red, respectively). The black line shows the $\boldsymbol{E} \times \boldsymbol{B}$ drift path of a zero energy particle during the reconfiguration. (Right top) Electric field and (right bottom) $\boldsymbol{E} \times \boldsymbol{B}$ drift speed versus time. The time scale of the reconfiguration is set to $20 \mathrm{~s}$.

\section{Ion dynamics during compression}

To illustrate the behavior of ions during compression of Mercury's magnetosphere, Fig. 3 presents model trajectories of different ion species (from top to bottom: $\mathrm{Na}^{+}, \mathrm{H}^{+}$, and $\mathrm{Ca}^{+}$) originating from the planetary exosphere in the highlatitude dayside sector. These test ions were launched from the same location at distinct (color-coded) times before the onset of the magnetic transition. In the two left panels of Fig. 3, this onset is indicated by color-coded closed circles. Looking first at the top row of Fig. 3 that relates to $\mathrm{Na}^{+}$, it can be seen in the left panels that, after convection over the polar cap into the nightside sector, the test ions intercept the field reversal at equator where their motion departs from a regular helical motion. This nonadiabatic behavior can be further appreciated in the right panel of Fig. 3 that displays a large magnetic moment increase near the end of the ion trajectories (coded in blue and green). During this nonadiabatic sequence that is due to the small spatial scale of the magnetic field as compared to the ion Larmor radius (e.g., Speiser, 1965; Chen and Palmadesso, 1986; Büchner and Zelenyi, 1989), $\mathrm{Na}^{+}$ rapidly travel westward (second panel from left) and are subsequently lost into the dusk magnetopause. This westward transport along the dawn-to-dusk electric field goes together with ion energization up to several keVs.

A feature of particular interest in the top row of Fig. 3 is that obtained for $\mathrm{Na}^{+}$located at mid-altitudes in the lobe at the reconfiguration onset (trajectories coded in green and yellow). For these ions, an increase of magnetic moment (first adiabatic invariant, denoted by $\mu$ hereinafter) by about one order of magnitude can be seen on the time scale of the magnetic transition. This contrasts with the behavior obtained for $\mathrm{Na}^{+}$located at low altitude (trajectory coded in red) or 

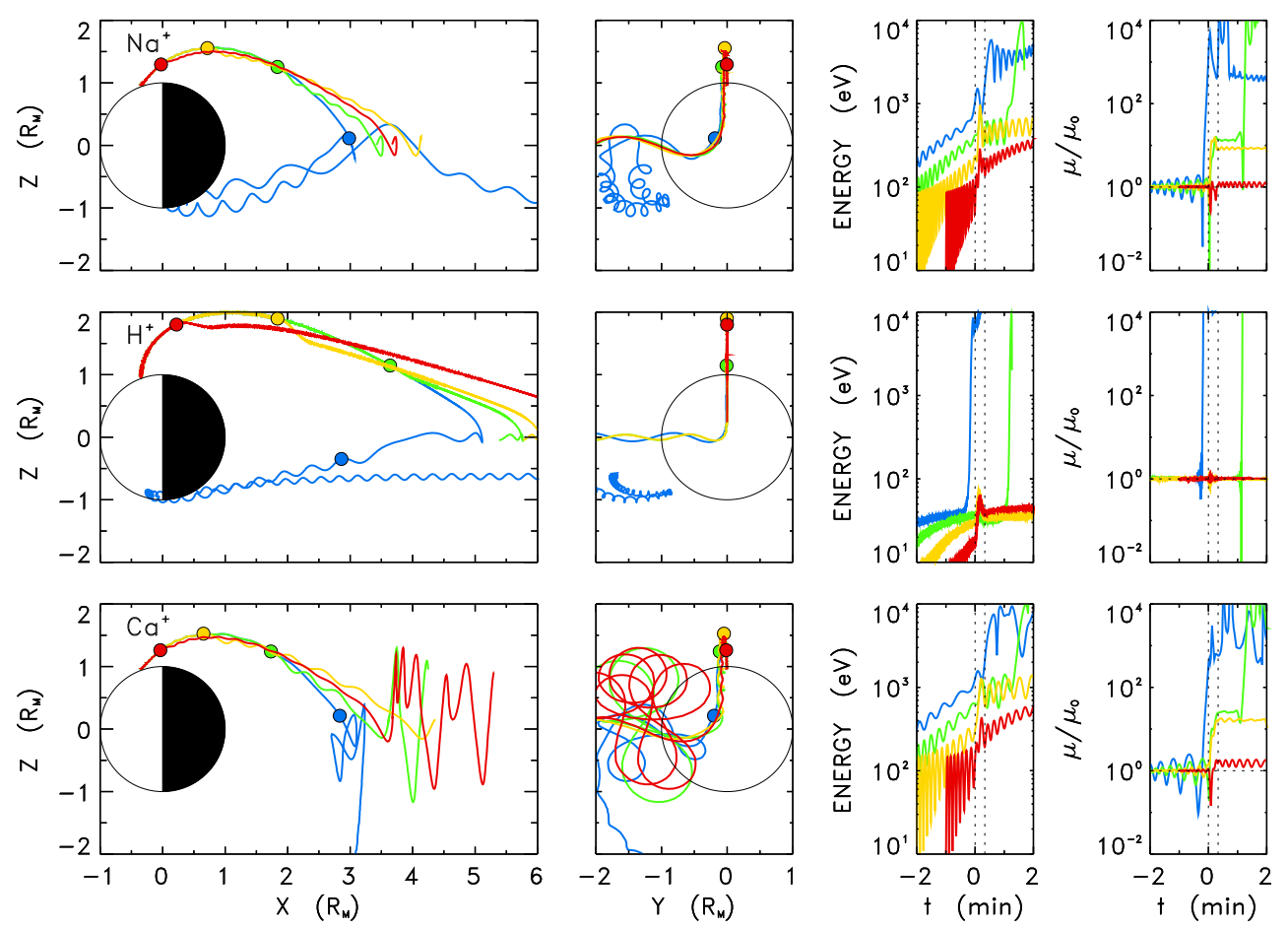

Fig. 3. Model ion trajectories during a 20-s magnetic transition: (from top to bottom) $\mathrm{Na}^{+}, \mathrm{H}^{+}$, and $\mathrm{Ca}^{+}$ions. (From left to right) Trajectory projections in the $\mathrm{X}-\mathrm{Z}$ and $\mathrm{Y}-\mathrm{Z}$ planes, energy, and magnetic moment (normalized to the initial value) as a function of time (measured from the reconfiguration onset). The different colors correspond to distinct times (by steps of $1 \mathrm{~min}$ ) of ejection in the high-latitude dayside sector. The distinct ion species are initialized at $70^{\circ}$ latitude on the noon meridian with similar velocities so that they are located in similar regions of space at the onset of the magnetic field reconfiguration. Color-coded closed circles in the two left panels show the position of the particles at this very onset. Vertical dotted lines in the right panels delimit the 20 -s magnetic transition.

in the equatorial region (trajectory coded in blue) at onset. This $\mu$ increase goes together with energization up to several hundreds of eVs (second panel from right).

For comparison, the second row of Fig. 3 shows the results obtained for protons launched from a similar location with similar time delays before onset. After convection over the polar cap into the nightside sector, these test protons also are subjected to nonadiabatic motions upon crossing of the field reversal at equator (in the rightmost panel, note the large $\mu$ variations obtained for the trajectories coded in blue and green). However, it is of interest to note that, unlike $\mathrm{Na}^{+}$, the magnetic moment of $\mathrm{H}^{+}$located at mid-altitudes in the lobe (trajectories coded in green and yellow) does not change during the magnetic transition. As far as energy is concerned (second panel from right), only a modest increase up to tens of eVs is obtained during the transition. This energization that occurs in an adiabatic manner likely follows from shortlived $\boldsymbol{E} \times \boldsymbol{B}$ related centrifugal acceleration as put forward by Cladis et al. (2000) during compression of the terrestrial magnetosphere. The comparison of top and middle rows in Fig. 3 thus suggests a mass selective ion energization during reconfiguration of the magnetic field lines. To confirm this outcome, the bottom row of Fig. 3 shows the results obtained for $\mathrm{Ca}^{+}$ions, using initial conditions similar to those of $\mathrm{Na}^{+}$and $\mathrm{H}^{+}$. In a like manner to $\mathrm{Na}^{+}$, it can be seen that

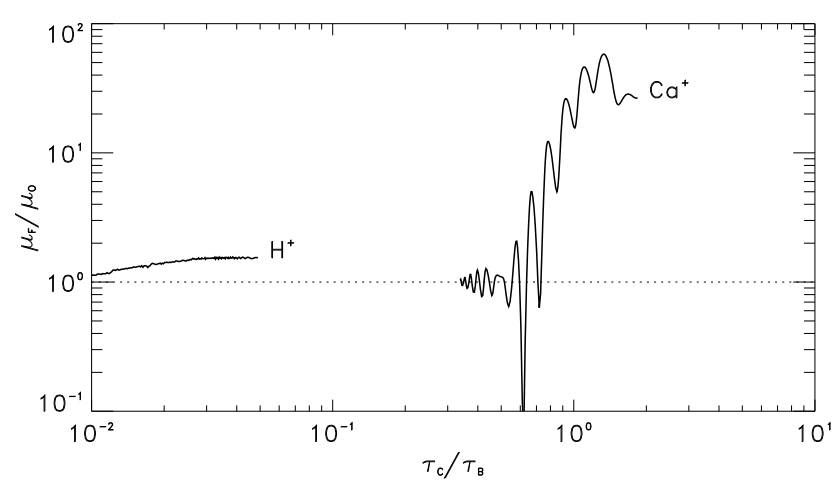

Fig. 4. Final magnetic moment (normalized to the initial value) of $\mathrm{H}^{+}$and $\mathrm{Ca}^{+}$ions as a function of cyclotron period-to-field variation time scale ratio.

$\mathrm{Ca}^{+}$located at mid-altitudes in the lobe at the reconfiguration onset (trajectories coded in green and yellow) are subjected to nonadiabatic heating, with $\mu$ increase by about one order of magnitude on the time scale of the magnetic transition.

To further investigate the above mass selective energization, Fig. 4 shows the $\mu$ changes obtained for ions with two widely different $m / q$ ratios ( $m$ being the ion mass, and $q$, its charge): $\mathrm{H}^{+}$and $\mathrm{Ca}^{+}$. These ions are located at different positions along the same flow path at the reconfiguration onset 

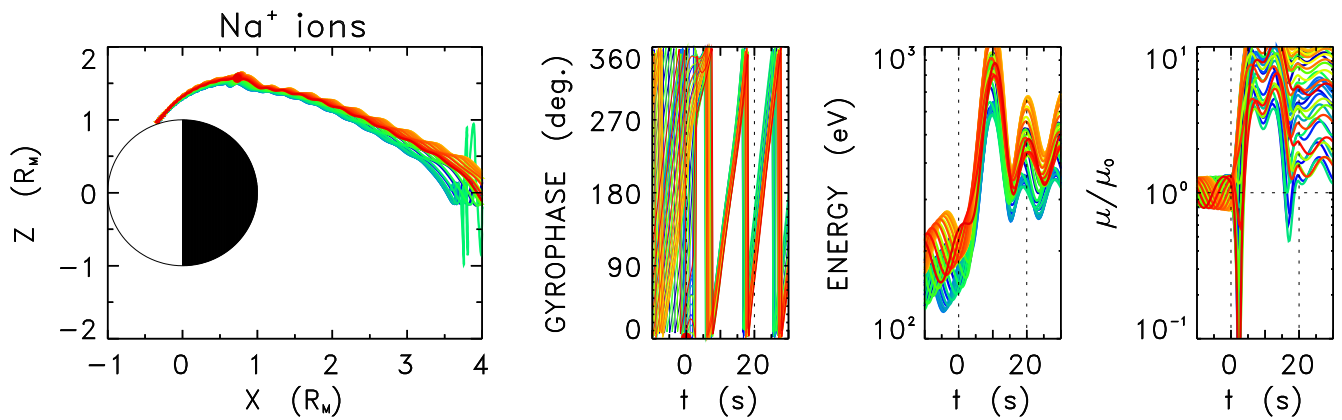

Fig. 5. Model trajectories of $\mathrm{Na}^{+}$ions during a 20-s magnetic transition: (from left to right) trajectory projection in the $\mathrm{X}-\mathrm{Z}$ plane, gyration phase, energy, and magnetic moment (normalized to the initial value) as a function of time (measured from the reconfiguration onset). The test ions are initialized at the same position in the high-latitude dayside sector. The different colors correspond to distinct initial phases of gyration (from $0^{\circ}$ to $360^{\circ}$ by steps of $10^{\circ}$ ).

and thus exhibit different cyclotron period-to-time scale ratios during the magnetic transition. In Fig. 4, the final magnetic moment (normalized to the initial one) is shown as a function of the $\tau_{C} / \tau_{B}$ ratio ( $\tau_{C}$ being the cyclotron period). It can be seen in this figure that, for protons traveling in the lobe, $\tau_{\mathrm{C}}$ is always much smaller than $\tau_{\mathrm{B}}$ and no significant $\mu$ change is obtained. In contrast, for $\mathrm{Ca}^{+}$ions, $\tau_{\mathrm{C}}$ is comparable to $\tau_{\mathrm{B}}$ and large $\mu$ enhancements (up to about two orders of magnitude) are obtained. Such a result suggests a resonant-like behavior during reconfiguration of the magnetic field lines. That is, for ions with $\tau_{\mathrm{C}}<\tau_{\mathrm{B}}$, the magnetic field does not change significantly during a cyclotron turn and the motion is adiabatic ( $\mu$ conserving) (e.g., Northrop, 1963). On the other hand, for ions with $\tau_{C}>\tau_{B}$, the work done by the induced electric field during a fraction of cyclotron turn is not significant enough to yield substantial perpendicular energization. In between, for ions with $\tau_{\mathrm{C}} \approx \tau_{\mathrm{B}}$, nonadiabatic behavior with possibly large $\mu$ enhancement is obtained due to phasing between the gyromotion and the surging electric field. This nonadiabatic energization is controlled by the magnitude of the peak induced electric field and thus depends upon $\Delta \boldsymbol{A}$ as well as $\tau_{\mathrm{B}}$, a shorter time scale of the magnetic transition resulting into a larger energy gain.

Because of phasing between the ion gyromotion and the surging electric field, one may expect specific gyrophase variations as well. This can be appreciated in Fig. 5 that shows the results obtained for $\mathrm{Na}^{+}$launched at the same time from the same position in the high-latitude dayside sector but with distinct phases of gyration. It can be seen in the rightmost panel of Fig. 5 that, on the whole, the test ions experience $\mu$ enhancement on the time scale of the magnetic transition; hence, large Larmor radii during their subsequent lobe transport (leftmost panel of Fig. 5). During the reconfiguration, the $\mathrm{Na}^{+}$energy increases up to several hundreds of eVs, as diplayed in the top row of Fig. 3. As for the gyration phase, it is apparent from the second panel at left in Fig. 5 that $\mu$ enhancements between $t=0$ and $t=20 \mathrm{~s}$ go together with prominent grouping of the particles. Such a bunching in gyration phase has been reported in a variety of studies dedicated to ion dynamics in the Earth's magnetotail (e.g., Ashour-Abdalla et al., 1993; Delcourt et al., 1996; Hoshino et al., 2000). In this region of space, breaking of adiabaticity and concurrent phase bunching occurs as a result of the sharp field reversal. The off-diagonal elements of the pressure tensor that follow from the ion phase coherence have been invoked to maintain stress balance in the magnetotail without requiring a large pressure gradient in the $\mathrm{X}$ direction such that $\nabla P=\boldsymbol{J} \times \boldsymbol{B}$. The results presented in Fig. 5 suggests that, at Mercury, nongyrotropy possibly plays a role also at high latitudes in the magnetospheric lobes if these are loaded with large $m / q$ material of planetary origin. Here, the build-up of nongyrotopic ion distributions may favor the growth of a variety of instabilities as well.

\section{Discussion}

To provide a more general view of nonadiabatic ion energization during compression of Mercury's magnetosphere, Fig. 6 shows the results of systematic trajectory computations for $\mathrm{Na}^{+}$and $\mathrm{H}^{+}$originating from the planetary exosphere in the high-latitude dayside sector. From left to right, Fig. 6 presents the color-coded energy of these particles at selected times of the reconfiguration process. In the initial state (leftmost panels of Fig. 6), $\mathrm{Na}^{+}$ions exhibit characteristic energies up to a few hundreds of $\mathrm{eV}$ in the magnetospheric lobes, while those of protons do not exceed $\sim 100 \mathrm{eV}$. This energization likely follows from $\boldsymbol{E} \times \boldsymbol{B}$ related centrifugal acceleration. In steady state, it is more pronounced at Mercury than at Earth because of smaller spatial scales (Delcourt et al., 2002). It is also apparent from Fig. 6 that, after transport through the lobes, both $\mathrm{Na}^{+}$and $\mathrm{H}^{+}$are subjected to nonadiabatic motion in the magnetotail current sheet with energization up to several $\mathrm{keVs}$; hence, the formation of a plasma sheet-like layer of energetic ions. Moving from left to right in the bottom panels of Fig. 6, it can be seen that, in the course of the magnetic transition, the average energy of 

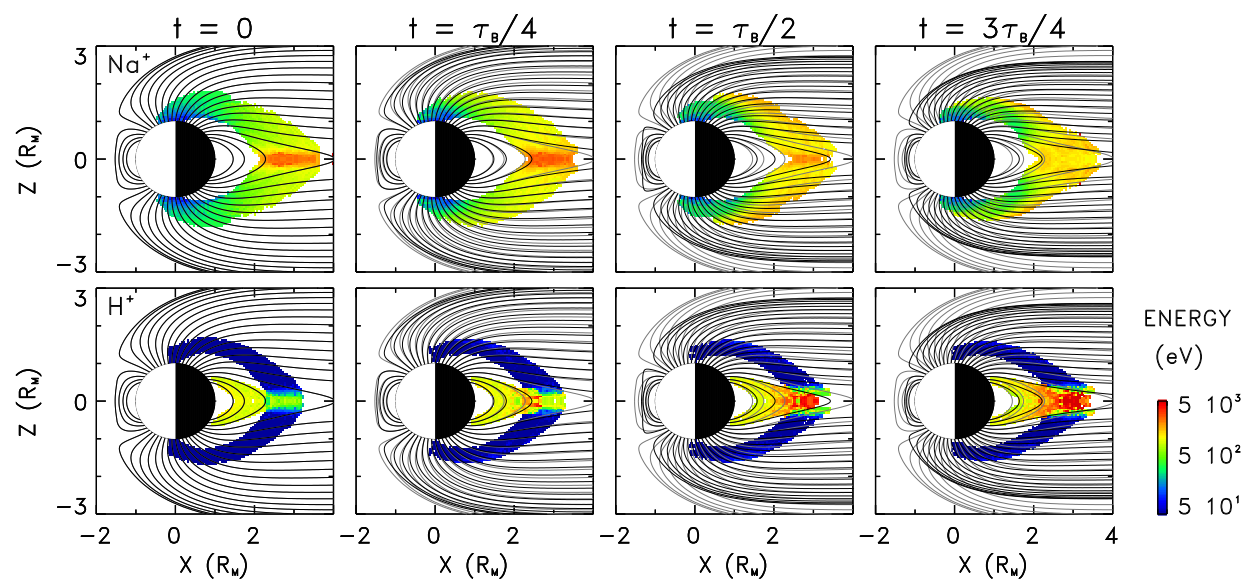

Fig. 6. Color-coded energy of (top) $\mathrm{Na}^{+}$ions and (bottom) protons in the $\mathrm{X}-\mathrm{Z}$ plane during a 20-s magnetic transition. The ions are launched from the high-latitude dayside sector. The panels from left to right show snapshots in the noon-midnight meridian plane at distinct times of the reconfiguration. Black and grey lines show the magnetic field lines at the corresponding time and in the initial configuration, respectively.

the proton population in the magnetospheric lobes does not significantly vary. On the whole, it does not exceed $100 \mathrm{eV}$. On the other hand, at equator, because of increasing elongation of the magnetic field lines, the local dynamical regime of protons gradually changes. Making use of the adiabaticity parameter $\kappa$ defined as the square root of the minimum curvature radius-to-maximum Larmor radius ratio (Büchner and Zelenyi, 1990), this regime evolves toward smaller $\kappa$ values or, equivalently, toward the quasi-adiabatic limit with predominant Speiser (1965) motions.

The comparison of bottom and top panels in Fig. 6 reveals a quite different response of $\mathrm{Na}^{+}$to compression of Mercury's magnetosphere. Indeed, it can be seen that, in the lobes, the average $\mathrm{Na}^{+}$energy rapidly increases from a few hundreds of eV up to a few keVs with a peak energy realized at mid-altitudes in the high-latitude nightside sector. It should be stressed here that this process differs from the adiabatic one reported by Cladis et al. (2000) that relates to a transient $\boldsymbol{E} \times \boldsymbol{B}$ related centrifugal acceleration. As discussed above (Fig. 4), the present energization occurs in a nonadiabatic manner and follows from resonance between the induced electric field and the ion gyromotion. As evidenced from Fig. 6, this process occurs well within the time scale of the magnetic transition. The net energization rate achieved strongly depends upon the amplitude and time scale of the reconfiguration. In this regard, similar trajectory computations using a smaller time scale ( $10 \mathrm{~s}$ instead of $20 \mathrm{~s}$ for the local reconfiguration of the magnetic field) reveal substantially larger energy gains (possibly up to $\sim 10 \mathrm{keV}$ ). In this latter case, the condition $\tau_{\mathrm{C}} \approx \tau_{\mathrm{B}}$ also is realized at somewhat smaller altitudes.

It is apparent from Fig. 5 that, due to magnetic moment enhancement at mid-altitudes, particles exhibit larger Larmor radii during their subsequent lobe transport. Accordingly, they approach the equatorial region in a less collimated man-
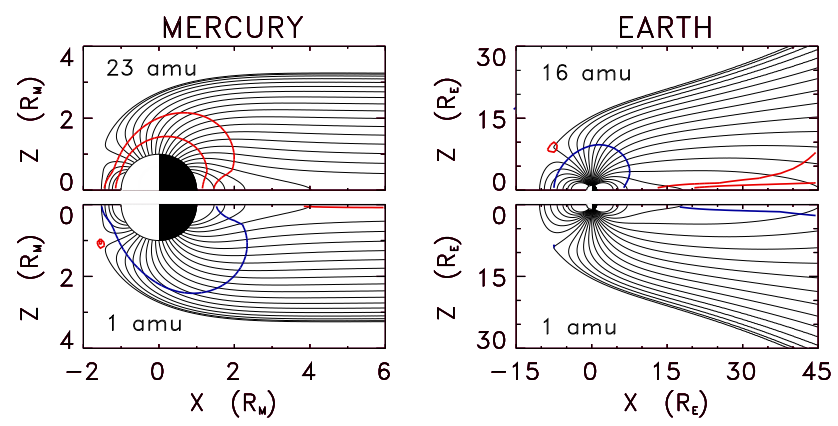

Fig. 7. Ion cyclotron period $\left(\tau_{\mathrm{C}}\right)$ normalized to the time scale $\left(\tau_{\mathrm{B}}\right)$ of the local magnetic field variation in the noon-midnight meridian plane (left) at Mercury and (right) at Earth. The top left panel relates to $\mathrm{Na}^{+}$ions, the top right panel to $\mathrm{O}^{+}$, the bottom panels to protons. The red contours correspond to $\tau_{\mathrm{C}} / \tau_{\mathrm{B}}=1$ and $\tau_{\mathrm{C}} / \tau_{\mathrm{B}}=2$, the blue contours, to $\tau_{\mathrm{C}} / \tau_{\mathrm{B}}=0.1$.

ner than in the initial steady state where they are subjected to pitch angle folding from low to high altitudes under $\mu$ conservation; hence, a different dynamical regime (e.g., quasitrapped as opposed to Speiser-type) and possibly weaker energization upon current sheet crossing. Still, on the whole, the short-lived nonadiabatic energization portrayed in Fig. 6 does not significantly affect the Dungey cycle time identified as that required for particles to drift from noon to midnight over the polar cap (e.g., Slavin et al., 2009) that is of the order of several minutes at Mercury (e.g., Delcourt et al., 2003).

A direct consequence of $\mu$ enhancement for $\tau_{\mathrm{C}} \approx \tau_{\mathrm{B}}$ in Fig. 4 is that nonadiabatic heating is expected to occur in distinct regions of space for different ion species (different $m / q$ ratios). To better illustrate this, the left panels of Fig. 7 show contours of the $\tau_{\mathrm{C}} / \tau_{\mathrm{B}}$ ratio for $\mathrm{Na}^{+}$(top) and $\mathrm{H}^{+}$(bottom). Looking at the $\tau_{\mathrm{C}} / \tau_{\mathrm{B}}=1$ and $\tau_{\mathrm{C}} / \tau_{\mathrm{B}}=2$ contours (red lines), it can be seen in the top left panel of Fig. 7 that, in 
the simple magnetic field transition considered in the present study, nonadiabatic heating of $\mathrm{Na}^{+}$ions is expected at midaltitudes both in dayside and nightside sectors. At these altitudes, protons are transported adiabatically (blue contour in bottom left panel of Fig. 7), their nonadiabatic regime being confined to the very vicinity of the magnetotail current sheet. This situation differs from that obtained at Earth, as can be seen by comparison of left and right panels in Fig. 7. Indeed, the right panels show $\tau_{\mathrm{C}} / \tau_{\mathrm{B}}$ contours for both $\mathrm{O}^{+}$(top) and $\mathrm{H}^{+}$(bottom) in the Tsyganenko (1989) model of the terrestrial magnetosphere $(\mathrm{Kp}=2)$, assuming a typical time scale of magnetic field variation of $2 \mathrm{~min}$. In this latter case, it can be seen that the nonadiabatic domain (red lines) for protons as well as for heavy ions is restricted to the equatorial magnetotail.

\section{Conclusions}

Because of the weak planetary magnetic field, the magnetosphere of Mercury is very dynamical and possibly subjected to prominent compression during events of enhanced solar wind pressure. The single-particle simulations performed provide some insights into the response of magnetospheric ions during such events. The simulations reveal that, under the effect of the electric field induced by the time-varying magnetic field, significant ion energization may be achieved in regions where the cyclotron period is of the order of the field variation time scale. This energization occurs in a nonadiabatic manner, being characterized by prominent magnetic moment enhancement and bunching of the particles in gyration phase. At Mercury, the above condition $\tau_{\mathrm{C}} \approx \tau_{\mathrm{B}}$ for nonadiabatic transport may be achieved in a wide range of altitudes and latitudes. This situation contrasts with that prevailing at Earth where $\tau_{\mathrm{C}} \approx \tau_{\mathrm{B}}$ is essentially realized in the equatorial magnetotail. Heavy ions originating from the planetary exosphere may be subjected to a rapid nonadiabatic energization during compression of Mercury's magnetosphere, which may lead to an abrupt loading of the magnetospheric lobes with energetic material.

Topical Editor R. Nakamura thanks A. Milillo and another anonymous referee for their help in evaluating this paper.

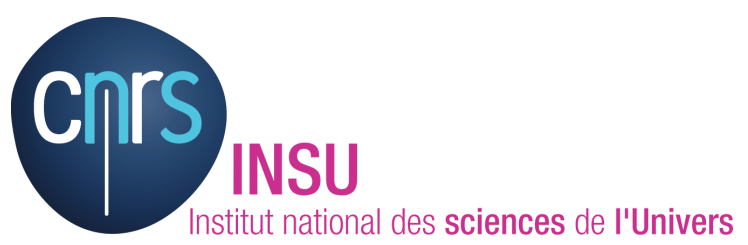

The publication of this article is financed by CNRS-INSU.

\section{References}

Anderson, B. J., Acuña, M. H., Korth, H., Purucker, M. E., Johnson, C. L., Slavin, J. A., Solomon, S. C., and McNutt Jr., R. L.: The structure of Mercury's magnetic field from MESSENGER's first flyby, Science, 321, 82-85, 2008.

Ashour-Abdalla, M., Zelenyi, L. M., Peroomian, V., and Richard, R. L.: On the structure of the magnetotail current sheet, Geophys. Res. Lett., 20, 2019-2222, 1993.

Benna, M., Anderson, B. J., Baker, D. N., Boardsen, S. A., Gloeckler, G., Gold, R. E., Ho, G. C., Killen, R. M., Korth, H., Krimigis, S. M., Purucker, M. E., McNutt Jr., R. L., Raines, J. M., McClintock, W. E., Sarantos, M., Slavin, J. A., Solomon, S. C., and Zurbuchen, T. H.: Modeling of the magnetosphere of Mercury at the time of the first MESSENGER flyby, Icarus, doi:10.1016/j.icarus.2009.11.036, available online: http://www. sciencedirect.com/, 2010.

Büchner, J. and Zelenyi, L. M.: Regular and chaotic charged particle motion in magnetotaillike field reversals: 1. Basic theory of trapped motion, J. Geophys. Res., 94, 11821-11842, 1989.

Chen, J. and Palmadesso, P. J.: Chaos and nonlinear dynamics of single-particle orbits in a magnetotaillike magnetic field, J. Geophys. Res., 91, 1499-1508, 1986.

Cladis, J. B., Collin, H. L., Lennartsson, O. W., Moore, T. E., Peterson, W. K., and Russell, C. T.: Observations of centrifugal acceleration during compression of magnetosphere, Geophys. Res. Lett., 27, 915-918, 2000.

Delcourt, D. C., Grimald, S., Leblanc, F., Berthelier, J.-J., Millilo, A., Mura, A., Orsini, S., and Moore, T. E.: A quantitative model of the planetary $\mathrm{Na}^{+}$contribution to Mercury's magnetosphere, Ann. Geophys., 21, 1723-1736, doi:10.5194/angeo-211723-2003, 2003.

Delcourt, D. C., Moore, T. E., Orsini, S., Millilo, A., and Sauvaud, J.-A.: Centrifugal acceleration of ions near Mercury, Geophys. Res. Lett., 29, p. 32,, 2002.

Delcourt, D. C., Belmont, G., Sauvaud, J.-A., Moore, T. E., and Martin Jr., R. F.: Centrifugally driven phase bunching and related current sheet structure in the near-Earth magnetotail, J. Geophys. Res., 101, 19839-19848, 1996.

Delcourt, D. C., Sauvaud, J.-A., and Pedersen, A., Dynamics of single-particle orbits during substorm expansion phase, J. Geophys. Res., 95, 20853-20865, 1990.

Heikkila, W. J. and Pellinen, R. J., Localized induced electric field within the magnetotail, J. Geophys. Res., 82, 1610-1614, 1977.

Hoshino, M., Mukai, T., Nishida, A., Kokubun, S., and Yamamoto, T.: Nongyrotropic ions as evidence for an X-type neutral region, Adv. Space Res., 26, 425-430, 2000.

Ip, W.-H.: Dynamics of electrons and heavy ions in Mercury's magnetosphere, Icarus, 71, 441-447, 1987.

Kabin, K., Heimpel, M. H., Rankin, R., Aumou, J. H., GomezPerez, N., Paral, J., Gombosi, T. I., Zurbuchen, T. H., Koehn, P. L., and DeZeeuw, D. L.: Global MHD modeling of Mercury's magnetosphere with applications to the MESSENGER mission and dynamo theory, Icarus, 195, 1-15, 2008.

Kabin, K., Gombosi, T. I., De Zeeuw, D. L., and Powell, K. G.: Interaction of Mercury with the solar wind, Icarus, 143, 397406, 2000.

Luhmann, J. G. and Friesen, L. M.: A simple model of the magnetosphere, J. Geophys. Res., 84, 4405-4408, 1979. 
Northrop, T. G.: The adiabatic motion of charged particles, Wiley Interscience, New York, 1963.

Potter, A. E. and Morgan, T. H.: Potassium in the atmosphere of Mercury, Icarus, 67, 336-340, 1986.

Potter, A. E. and Morgan, T. H.: Discovery of sodium in the atmosphere of Mercury, Science, 229, 651-653, 1985.

Russell, C. T. and Walker, R. J.: Flux transfer events at Mercury, J. Geophys. Res., 90, 11067-11074, 1985.

Slavin, J. A., Acuña, M. H., Anderson, B. J., Baker, D. N., Benna, M., Boardsen, S. A., Gloeckler, G., Gold, R. E., Ho, G. C., Korth, H., Krimigis, S. M., McNutt Jr., R. L., Raines, J. M., Sarantos, M., Schriver, D., Solomon, S. C., Trávniček, P., and Zurbuchen, T. H.: MESSENGER observations of magnetic reconnection in Mercury's magnetosphere, Science, 324, 606-610, 2009.
Slavin, J. A., Acuna, M. H., Anderson, B. J., Baker, D. N., Benna, M., Gloeckler, G., Gold, R. E., Ho, G. C., Killen, R. M., Korth, H., Krimigis, S. M., McNutt Jr., R. L., Nittler, L. R., Raines, J. M., Schriver, D., Solomon, S. C., Starr, R. D., Trávniček, P., and Zurbuchen, T. H.: Mercury's magnetosphere after MESSENGER's first flyby, Science, 321, 85-89, 2008.

Speiser, T. W.: Particle trajectory in model current sheets, 1, Analytical solutions, J. Geophys. Res., 70, 4219-4226, 1965.

Tsyganenko, N. A.: Magnetospheric magnetic field model with a warped tail current sheet, Planet. Space Sci., 37, 5-20, 1989.

Volland, H.: A model of the magnetospheric convection electric field, J. Geophys. Res., 83, 2695-2699, 1978.

Zurbuchen, T. H., Raines, J. M., Gloeckler, G., Krimigis, S. M., Slavin, J. A., Koehn, P. L., Killen, R. M., Sprague, A. L., McNutt Jr., R. L., and Solomon, S. C.: MESSENGER observations of the composition of Mercury's ionized exosphere and plasma environment, Science, 321, 90-92, 2008. 